\title{
Learning of letter pairs as a prototype of first language learning'
}

ERWIN M. SEGAL AND TERRY G. HALWES

ARLINGT'ON STATE COLLEGF.

\begin{abstract}
Abstraet
Twenty-four letter pairs generated by elementary grammars formally similar to a child's first grammars were presented to 3 groups of adult Ss for recall. The first grammar had a simple structure, the second a more complex structure, and the third, random pairings. In all of these groups, the Ss responded as if they had been presented the simple grammar. They learned neither the pairs nor their underlying structure.

\section{Problem}

Smith (1963) investigated certain structural aspects involved in the learning of simple verbal sequences. He constructed letter pairs from two classes of letters, $A$ and $B$, with the A-letters appearing first in all sequences, and the B-letters appearing second, and presented them to Ss for recall. He generated pairs from a vocabulary of five A-letters and five B-letters. Five of the possible $\mathrm{AB}$ pairs were not presented. To another group of Ss he presented 20 pairs randomly constructed from the 10 letters. He found that Ss learned the $A B$ pairs which were not presented. They appeared as intrusions far too frequently to be explained as guesses or random errors.

The Smith study demonstrates a technique which may be used to investigate many grammatical phenomena, and his results lead to further speculation on grammatical learning. Consider the child and grammatical phenomena as they develop. The first sentences that children learn beyond single word utterances consist of two words (Brown \& Fraser, 1963; Braine, 1963). One may classify the two words into two sets. One set seems to be closed, and the words in it may be called pivot words (Braine, 1963). The other set is an open nounlike set. For some children a word from the open set tends to come first followed by a word from the closed set, and for some children the situation is reversed. However, for many children, one may formalize the first grammar as "Utterance $\rightarrow A+B, A \rightarrow a_{1}, \ldots a_{i}, B \rightarrow b_{1}, \ldots b_{j}$." This grammar is essentially similar to the grammar that Smith (1963) used in his study.

By analyzing the child's grammar further, it can be seen that in many cases the next phase of grammatical development is the adding of an optional sentence form. The child learns a new set of pivot words, and these may appear on the other side of the open class. However, when these words appear the other pivot words do not. The grammar now reads, "U $\rightarrow S, S \rightarrow A+B$, $\mathrm{S} \rightarrow \mathrm{B}+\mathrm{C}$, etc." Each sentence now contains a member of the open class B, and a member of one of the pivot classes, but not both.

Children seem to learn complexgrammars by stages.
They start with utterances which may be described by very simple reduced grammars, and as they get older their grammars get more complex (Brown \& Fraser, 1963). This experiment was designed as an attempt to reproduce some of the developmental aspects of grammatical learning by using adult Ss and miniature languages.

We presented to each of three groups of Ss a series of letter pairs. For one group the pairs were generated by a grammar of the form, "S $\rightarrow \mathrm{A}+\mathrm{B}$." The grammar is formally equivalent to the one used by Smith, and also to the first grammar of children. For a second group, pairs were presented which were generated by a grammar with two basic rules, "S $\rightarrow A+B$ " and "S $\rightarrow \mathrm{B}+\mathrm{C}$. " This grammar is formally equivalent to the second language children learn. The third group was given random groups of letter pairs. We predicted that the Ss would learn the grammars by stages. They would all first learn the vocabulary, then the experimental groups would generate pairs derived by the elementary grammar, and the second experimental group would restrict the pairs to those generated by the more complex grammar.

\section{Method}

Three groups of 20 introductory psychology students were run in groups of from 2 to 7 Ss. Twenty-four letter pairs were presented orally in random order at a 3 sec.inter-pair interval. After the presentation $\mathrm{S}$ was given $2 \mathrm{~min}$. to write all of the pairs that he could recall. The procedure was repeated for a total of 12 trials.

The letter pairs for all three groups were generated from the same set of 12 consonants. For Group I (GI), six of the letters were randomly assigned to class $\mathrm{A}$ and six to class $\mathrm{B}$. Of the 36 possible $\mathrm{AB}$ pairs, 24 were presented. For Group II (GII), 4 of the letters were assigned to class $A, 4$ to class $B$, and 4 to class $C$. AB and $B C$ pairs were generated, and 24 of the possible 32 pairs were presented. For Group III (GIII), 24 pairs were constructed randomly from the 12 letters with the constraint that all letters must appear twice in both the first and last position and no single letter could appear twice in a given pair.

\section{Results}

There were no major differences in the rate of learning the pairs for the three groups. The between groups analysis of variance for total number of pairs recalled accurately was not significant, $F(2,57)=2.61, p>.10$. The trials by treatment interaction was barely significant, $F(22,62)=1.61, p<.05$. GI performed slightly better than GII, which performed slightly better than GIII.

During recall, Ss, make many errors of commission. An analysis of these intrusions gives clues to the learning process. If the structural relationships within each set of presented pairs were basically irrelevant and intrusions were made by idiosyncratic pairings of letters, the intrusion data for the three groups should be similar. The number of intrusions for the three groups was analyzed and the differences between groups and 
the groups by trials interaction were both insignificant, p>.10. However, there was a significant trial effect, $F(1,418)=2.28, p<.02$, which was primarily due to an increase in the number of intrusions over trials, $\mathrm{F}(1,418)=22.40, \mathrm{p}<.001$.

The theoretical grammars were also investigated by use of the intrusion data. Nonpresented pairs which could be generated by different grammars were compared over trials for their relative rate of occurrence in the response protocols. A comparison of the different classes of intrusions per opportunity is given in Fig. 1.

The intrusion rates for the same four nonpresented pairs were compared for the three groups. The pairs were generated by the grammars of both Groups I and II. These pairs were emitted at vastly different rates by the different groups, $F(2,57)=13.31, p<.001$. (The mean number of pairs emitted over 12 trials for GI = 10.4, for $\mathrm{GII}=4.9$, and for $\mathrm{GIII}=0.95$.) All between groups differences were significant, $\mathrm{p}<.05$. These results show that specific intrusions are conditioned by the environment in which they are emitted.

For GI the grammatical intrusions (nonpresented AB pairs) were weighted ${ }^{2}$ and compared against nongrammatical intrusions (other pairs whose component letters were in the 12 letter vocabulary). There was a highly significant difference in types of intrusions, $F(1,19)=$ $18.98, \mathrm{p}<.001$; a significant trials effect, $F(11,209)=$ $3.26, \mathrm{p}<.001$; and a significant type by trials interaction, $F(11,209)=2.94, p<.01$. The grammatical intrusions occurred more often and increased more over trials.

For GII an equivalent analysis was run. However, the intrusions were divided into three classes: (1) nongrammatical pairs, (2) semi-grammatical pairs (AC or BB pairs. The AC and BB pairs are generated by a simple two class grammar; see discussion.), and (3) grammatical pairs (nonpresented AB or BC pairs). Again the types of intrusions were highly significant, $F(2,38)=$ $23.1, \mathrm{p}<.001$. Unexpectedly, there were no significant trials or interaction effects. Orthogonal comparisons showed that the significant differences in types of intrusions was entirely due to the grammaticalnongrammatical contrast, $F(1,38)=46.0, p<.001$. There were no significant differences between weighted scores of semi-grammatical and grammatical pairs, $F=0.12$.

\section{Diseussion}

What is it that $\mathrm{S}$ learns? He does not simply learn the presented pairs by rote. If he did so, one would expect the number of S's emissions of presented pairs to increase and nonpresented pairs to decrease over trials (Braine, 1965; Gough \& Segal, 1965). Assume S generated pairs according to rules. From a functional and pragmatic point of view, one expects $S$ to learn rules which would enable him to generate many presented pairs and few, if any, nonpresented pairs. Then one may expect $\mathrm{S}$ to learn rules which exclude the nonpresented pairs. If the number of generation rules, plus the exclusion rules, is much greater than the number of strings to be learned, and $S$ is learning slowly, then one might expect $\mathrm{S}$ to change his strategy, by either

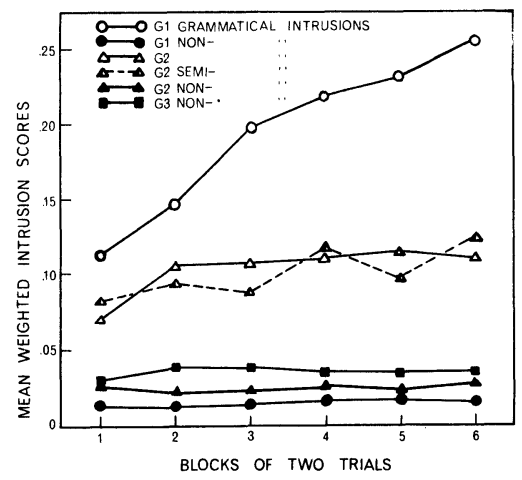

Fig. 1. A comparison of the different classes of intrusions among the three experimental groups.

learning new rules or switching to rote learning.

It seems to be true that $\mathrm{S}$ generates pairs according to rules, but in this study it is not true that Ss learned rules which generated few, if any, nonpresented pairs. Also, it seems that S never modified his rules and continued to generate an ever increasing number of nonpresented pairs over the 12 trials of the experiment. $S$ did not behave very pragmatically, and he could not learn 24 pairs of letters in 12 trials.

The Ss in each of the three groups performed differ ently in some respects, but what they learned was similar. $\mathrm{S}$ learned there were 2 letters in each sequence. The instructions contained this information. He learned approximately the number of pairs presented. S learned the set of letters which occurred first in the sequence and the set of letters which occurred second, independently of each other. He learned to concatenate members of these two sets of events. For Group I the sets each contained 6 letters; for Group II the sets each contained 8 letters, including 4 occurring in both sets; and for Group III the sets each contained 12 letters, all of which appear in both sets. The grammar invented by $\mathrm{S}$ contains these rewrite rules: " $\mathrm{S} \rightarrow \mathrm{P} ; \mathrm{P} \rightarrow \mathrm{A}+\mathrm{B}$; $A \rightarrow a_{1}, \ldots a_{i} ; B \rightarrow b_{1}, \ldots b_{j}$ " $S$ then generated pairs and attempted to recognize them as pairs which were presented.

\section{References}

Braine, M. D. S. The ontogeny of English phase structure: The First Phase. Language, 1963, 38, 1-13.

Braine, M. D. S. The insufficiency of a finite state model for verbal reconstructive memory. Psychon. Sci., 1965, 2, 291-292.

Brown, $\mathbf{R}, \&$ Fraser, C. The acquisition of syntax. In C. N. Cofer \& Barbara S. Musgrave (Eds.), Verbal behavior and learning. New York: McGraw-Hill, 1963.

Gough, P. B., \& Segal, E. M. Comment on “The insufficiency of a finite state model of verbal reconstructive memory." Psychon. Sci., 1965, 3, 155-156.

Smith, K. H. Recall of paired verbal units under various conditions of organization. Unpublished doctoral dissertation, University of Minnesota, 1963.

\section{Notes}

1. This paper was supported in part by USPHS Research Grant MH 10042-01 to the senior author

2. The intrusion scores were computed by taking the number of intrusions in a class and dividing it by the total number of letter combinations in that class. 